\title{
AN IMPROVED STABILITY RESULT FOR RESONANCES
}

\author{
BY
}

MARK S. ASHBAUGH AND CARL SUNDBERG

\begin{abstract}
We prove stability of shape resonances for the sequence of Schrödinger equations $\left(-d^{2} / d x^{2}+U(x)+W_{n}(x)\right) \psi(x)=E \psi(x), 0 \leqslant x<\infty$, in the limit $n \rightarrow$ $\infty$ where the barrier potentials $W_{n}(x)$ are integrable, nonnegative, supported in the interval $[1, a](1<a<\infty)$, and approach infinity pointwise a.e. for $x \in[1, a]$ as $n \rightarrow \infty$. In the course of our investigation we prove that for suitable complex initial conditions the solution to the Riccati equation $S^{\prime}(x)=1-\left(W_{n}(x)-E\right)[S(x)]^{2}$ goes to 0 as $n \rightarrow \infty$ uniformly on compact subsets of $[1, a]$. Our approach is via ordinary differential equations using outgoing wave boundary conditions to define resonances. Our stability result extends a similar result of Ashbaugh and Harrell, who use an argument based on asymptotics and the implicit function theorem to study the above problem with $\lambda V(x)$ replacing $W_{n}(x)$. Our approach is to use the Riccati equation analysis mentioned above and an application of Hurwitz's Theorem from complex variable theory.
\end{abstract}

1. Introduction. The behavior of eigenvalues of Schrödinger operators has been the subject of extensive investigation since the advent of quantum mechanics. In particular, perturbation theory has been on a firm mathematical footing since the work of Rellich in the thirties [5]; Kato [6] and Sz.-Nagy [7] also made important contributions. In contrast, the study of resonances has received comparatively little attention. Only rather recently have some of the most basic properties of eigenvalues been extended to resonances [8-10]. This disparity is not surprising in view of the relative difficulty of studying resonances in a Hilbert space framework. Whereas the eigenvalues of a Schrödinger operator can be dealt with directly in terms of the given selfadjoint operator, at best resonances can be viewed as eigenvalues of certain associated nonselfadjoint operators, a result established by Simon [8] for the case of dilation analytic potentials. In the one-dimensional or separable multi-dimensional case, however, one has at one's disposal techniques from the theory of ordinary differential equations. In this setting, a recent paper by Ashbaugh and Harrell [3] developed perturbation theory for shape resonances, i.e. resonances arising in two-body potential scattering from the shape of the potential. In the present paper we extend one of their most basic results, that of stability of resonances under perturbations. Other recent work on related problems using functional analytic methods is contained in [11-16 and 24].

Received by the editors February 15, 1983.

1980 Mathematics Subject Classification. Primary 81C12, 81F05; Secondary 34B05, 34B25.

Key words and phrases. Quantum mechanical resonances, stability of shape resonances, Riccati equations, outgoing wave boundary condition.

(C)1984 American Mathematical Society $0002-9947 / 84 \$ 1.00+\$ .25$ per page 
In general outline, we follow here the formulation of the resonance problem as set forth in Ashbaugh-Harrell. Since our primary interest is in three-dimensional problems with spherical symmetry we shall be concerned with the Schrödinger equation

$$
-\psi^{\prime \prime}(x)+V(x) \psi(x)=E \psi(x) \text { on }[0, \infty)
$$

with Dirichlet boundary condition at $x=0$ and with outgoing wave or GamowSiegert $[1,2]$ boundary condition for $x \rightarrow \infty$. In [3], this equation is considered for

$$
V(x)=U(x)+\lambda W(x)
$$

where $U$ is a piecewise continuous real-valued function on $(0,1]$, supported in $[0,1]$, and with $\lim _{x \downarrow 0} x^{2} U(x)=l(l+1), l$ a nonnegative integer. $W$ is taken to be piecewise continuous, supported in $[1, a]$ for some finite $a>1$, and strictly positive on $(1, a)$. Under these hypotheses the potential $V$ represents a barrier on the interval $(1, a)$ which can be scaled in height through the parameter $\lambda$. The authors then undertake a systematic study of the asymptotic behavior of the resonance energies in the $\lambda \rightarrow \infty$ limit. In particular, they develop formulas for the real and imaginary parts of the resonance energies which are asymptotically valid as $\lambda \rightarrow \infty$. These results should be viewed as perturbative results relating the behavior of resonances in the above problem to the eigenvalues of the unperturbed problem

$$
-\psi^{\prime \prime}(x)+U(x) \psi(x)=e \psi(x) \text { on }[0,1]
$$

with Dirichlet boundary conditions imposed at both endpoints; this unperturbed problem may be regarded as the $\lambda=\infty$ case of the above problem. More specifically, one can show that for any resonance energy $E(\lambda)$ such that $\lim _{\lambda \rightarrow \infty} E(\lambda)$ exists, this limit must be one of the $\lambda=\infty$ eigenvalues $e$. In fact, this result, which we call a stability result for resonances, is fundamental to the subsequent perturbative development.

In the present paper we establish a similar stability result in the following more general situation. We take

$$
V(x)=U(x)+W_{n}(x)
$$

in equation (1.1). Here $U$ is a real-valued function supported in $[0,1]$, integrable away from 0 , and satisfying $\lim _{x \downarrow 0} x^{2} U(x)=l(l+1), l$ a nonnegative integer. The $\left\{W_{n}\right\}_{n=1}^{\infty}$ are integrable real-valued nonnegative functions supported in $[1, a]$ such that $W_{n}(x) \rightarrow \infty$ as $n \rightarrow \infty$ for almost all $x \in[1, a]$. Thus the manner in which the potential barriers are allowed to approach infinity is more general in the present paper; note, in particular, that our barriers are not required to go to infinity monotonically. To prove stability of resonances under these weakened hypotheses, however, we cannot rely upon asymptotic formulas for the solutions to (1.1) with $V(x)$ given by (1.2) as was done in [3]. Instead, we will use various comparison arguments about such solutions which will allow us to reach the desired conclusion via a different route. Our methods yield some results about complex-valued solutions to a Riccati equation which may be of interest in their own right.

We turn now to casting our problem into the form in which we wish to view it for the remainder of the paper. First, we define two solutions, $\psi_{0}(x ; E, n)$ and 
$\psi_{\infty}(x ; E, n)$, to the differential equation (1.1) where now $V(x)$ is given by (1.4). $\psi_{0}$ is defined by specifying Dirichlet boundary conditions at $x=0$,

$$
\psi_{0}(0 ; E, n)=0 \text { and } \psi_{0}^{\prime}(0 ; E, n)=1,
$$

and $\psi_{\infty}$ is defined by specifying Gamow-Siegert boundary conditions as $x \rightarrow \infty$ which, because $V(x)=0$ for $x>a$, may be imposed at $x=a$ as

$$
\psi_{\infty}(a ; E, n)=1 \text { and } \psi_{\infty}^{\prime}(a ; E, n)=i \sqrt{E} \text {. }
$$

Our convention on the square root is that it be the positive square root on the positive real axis and that its branch cut lies along the negative real axis. Resonance energies are complex so this comment is vital. For definiteness of $\psi_{0}$ and $\psi_{\infty}$ these conditions fix normalization as well as the desired boundary condition-a simple Gamow-Siegert boundary condition would just require $\psi_{\infty}(x)=A e^{i \sqrt{E} x}$ for $x \geqslant a$. Note that $\psi_{0}$ is independent of $n$ for $x \in[0,1]$ and that it solves the differential equation (1.3) with $e=E$ since $W_{n}(x)=0$ on this interval. We shall use these facts and suppress the index $n$ in the following since we shall never evaluate $\psi_{0}$ for $x>1$. Resonance energies for (1.1) are determined as complex values $E$ such that $\psi_{0}(x ; E)$ and $\psi_{\infty}(x ; E, n)$ are linearly dependent. We state this as a definition.

DEFINITION 1.1. A resonance of (1.1) is a number $E \in \mathbf{C} \backslash(-\infty, 0]$ for which

$$
0=F_{n}(E) \equiv \frac{\psi_{0}(1 ; E)}{\psi_{0}^{\prime}(1 ; E)}-\frac{\psi_{\infty}(1 ; E, n)}{\psi_{\infty}^{\prime}(1 ; E, n)}=\frac{W\left\{\psi_{0}, \psi_{\infty}\right\}}{\psi_{0}^{\prime}(1) \psi_{\infty}^{\prime}(1)}
$$

Here $W\left\{\psi_{0}, \psi_{\infty}\right\} \equiv \psi_{0} \psi_{\infty}^{\prime}-\psi_{0}^{\prime} \psi_{\infty}$ is the Wronskian of $\psi_{0}$ and $\psi_{\infty}$. We take the following facts as well known $[17,18]$ :

(1) $\psi_{0}(x ; E)$ is analytic in $E$,

(2) $\psi_{\infty}(x ; E, n)$ is analytic in $E$ except at $E=0$, where it has a branch point due to the square root in (1.6).

Hence, $F_{n}(E)$ is an analytic function of $E$ for $E \in \mathbf{C} \backslash(-\infty, 0]$ except perhaps for poles at the zeros of $\psi_{0}^{\prime}(1 ; E)$ and $\psi_{\infty}^{\prime}(1 ; E, n)$. For our purposes these exceptions present no difficulties.

In $\S \S 2$ and 3 we show that

$$
\frac{\psi_{\infty}(1 ; E, n)}{\psi_{\infty}^{\prime}(1 ; E, n)} \rightarrow 0
$$

uniformly for $E$ in certain compact subsets of $\mathbf{C}$ as $n \rightarrow \infty$. We do this by analyzing the solutions to the Riccati equation satisfied by $\psi_{\infty} / \psi_{\infty}^{\prime}$. Thus our stability result will involve the zeros of

$$
F(E)=\lim _{n \rightarrow \infty} F_{n}(E)=\frac{\psi_{0}(1 ; E)}{\psi_{0}^{\prime}(1 ; E)} .
$$

These are precisely the zeros of $\psi_{0}(1 ; E)$, i.e. the eigenvalues of the unperturbed problem (1.3). In $\$ 4$ we use Hurwitz's Theorem $[19,20]$ to conclude that to each unperturbed eigenvalue $e>0$ there corresponds, for sufficiently large $n$, a unique nearby resonance energy $E_{n}$ with $E_{n} \rightarrow e$ as $n \rightarrow \infty$. 
2. Preliminary results. In this section we begin the ordinary differential equations arguments which lead to the result $\psi_{\infty}(1 ; E, n) / \psi_{\infty}^{\prime}(1 ; E, n) \rightarrow 0$ as $n \rightarrow \infty$. We are interested in the behavior of $\psi_{\infty}$ at 1 having prescribed its initial data at $x=a$. Here we address the analogous problem on $[0,1]$ where behavior at $x=0$ is prescribed and that at $x=1$ is to be determined. These problems are equivalent via a reflection and a rescaling of $x$. Moreover, since our interest lies in the ratio $\psi_{\infty} / \psi_{\infty}^{\prime}$ we focus attention on the Riccati equation which it satisfies. Here we concern ourselves with the following simplified Riccati equation which will be useful in subsequent comparison arguments:

$$
S^{\prime}(x)=1-A(x)[S(x)]^{2} \text { on }[0,1] .
$$

with $S(0)=c e^{i \theta}, c \geqslant 0, \theta \in[0, \pi / 2]$. In the following we shall always assume that $A(x)$ is integrable and nonnegative in $[0,1]$.

We begin our study of equation (2.1) by noting that we may treat it as a system of two differential equations for the real and imaginary parts of $S(x)=f(x)+i g(x)$ :

$$
\begin{gathered}
f^{\prime}(x)=1-A(x)\left[f(x)^{2}-g(x)^{2}\right], \\
g^{\prime}(x)=-2 A(x) f(x) g(x)
\end{gathered}
$$

with initial conditions $f(0)=c \cos \theta$ and $g(0)=c \sin \theta$. For the present, we assume that $f(x)$ and $g(x)$ exist on $[0,1]$. The possibility that they do not will be dispensed with following Lemma 2.4 to conclude the argument.

LEMMA 2.1. $f(x)>0$ for $x \in(0,1]$.

Proof. From equation (2.2a) we note that $f^{\prime}(x) \geqslant 1$ at zeros of $f(x)$. Hence, on any interval $f$ can be 0 at most once and must be negative to the left of such a zero and positive to the right. Since $f(0) \geqslant 0$ here, $f$ must be positive to the right of 0 .

LEMMA 2.2. Either $g(x) \equiv 0$ or $g(x)>0$ for $x \in[0,1]$; in addition, $g^{\prime}(x) \leqslant 0$ on this interval.

Proof. By the preceding lemma, we can assume that $f(x)$ is a known positive function. Then the differential equation $g^{\prime}=-2 A f g$ together with the initial condition $g(0)=c \sin \theta \geqslant 0$ gives the result, since the only solution to this differential equation which has zeros is the zero solution.

LEMMA 2.3. There is at most one point $x_{1} \in[0,1]$ at which $f=g$. For such a point, $f(x)<g(x)$ on $\left[0, x_{1}\right)$ and $f(x)>g(x)$ on $\left(x_{1}, 1\right]$.

Proof. Suppose that $x_{1} \in[0,1]$ has the property that $f\left(x_{1}\right)=g\left(x_{1}\right)$. Then $f^{\prime}\left(x_{1}\right)$ $=1$ whereas by the preceding lemma $g^{\prime}(x) \leqslant 0$ on $[0,1]$. Hence at any such point, $f$ must overtake $g$ and this can happen at most once.

LEMMA 2.4. $f(x) \leqslant 1+c$ on $[0,1]$.

Proof. If $f(x) \leqslant g(x)$ for all $x \in[0,1]$, then $f(x) \leqslant g(0)=c \sin \theta<1+c$ because $g$ is decreasing. If $f(a)=g(a)$ for some $a \in(0,1)$, then $f(x) \leqslant g(x) \leqslant c<1$ $+c$ for $x \leqslant a$. For $x>a$, Lemma 2.3 and equation (2.2a) imply that $f^{\prime}(x) \leqslant 1$ and 
hence $f(x)-f(a) \leqslant x-a \leqslant 1-a$. Then from $f(a)=g(a) \leqslant c$ we obtain $f(x) \leqslant 1$ $+c-a<1+c$. If $f(x)>g(x)$ on $[0,1]$, then again $f^{\prime}(x) \leqslant 1$ and hence $f(x) \leqslant 1$ $+c$.

REMARK. As proven above, Lemmas 2.1-2.4 hold only on an interval [ $0, b)$ where $b \in(0,1]$ because $f$ and $g$ may not be continuable past $b$. In this case, however, $f(x)$ must approach infinity as $x \uparrow b$ because of Lemmas 2.1 and 2.2, and this contradicts Lemma 2.4. This shows that $f$ and $g$ exist on $[0,1]$ and that, therefore, the above lemmas hold as stated.

Having discovered these crude qualitative features of the functions $f$ and $g$, we proceed to an investigation of how these solutions depend on $A$. Consider the family of Riccati equations

$$
S_{n}^{\prime}(x)=1-A_{n}(x)\left[S_{n}(x)\right]^{2}
$$

all with initial condition $S_{n}(0)=c e^{i \theta}, c \geqslant 0, \theta \in[0, \pi / 2]$, where the $A_{n}(x)$ are nonnegative real-valued integrable functions going to infinity pointwise almost everywhere in $[0,1]$ as $n \rightarrow \infty$. First we state a lemma concerning measurable functions which will be needed frequently in the proofs which follow. Here and in the sequel, if $F \subset \mathbf{R}$ is measurable then $|F|$ means the Lebesgue measure of $F$.

LEMMA 2.5. Let $E \subset \mathbf{R}$ be a measurable set having finite measure. Let $\left\{A_{n}\right\}_{n=1}^{\infty}$ be a sequence of measurable functions with $A_{n}(x) \rightarrow \infty$ for almost all $x \in E$. Then for each $K, \delta>0$ there exists a measurable set $P \subset E$ with $|P|<\delta$ and an integer $N$ such that for all $x \in E \backslash P$ and $n \geqslant N$ we have $A_{n}(x)>K$.

Proof. Standard, see Royden [23].

We continue now with our discussion of the $n \rightarrow \infty$ behavior of solutions to equation (2.3).

Proposition 2.6. Take $A_{n}(x), n=1,2,3, \ldots$, as above. Let $S_{n}(x)$ solve equation (2.3) with the given initial condition. Then $S_{n}(x)$ exists on $[0,1]$ and $S_{n}(x) \rightarrow 0$ as $n \rightarrow \infty$ uniformly for $c$ in compact subsets of $[0, \infty), \theta \in[0, \pi / 2]$, and $x$ in compact subsets of $(0,1]$.

Proof. We have shown that $S_{n}(x)=f_{n}(x)+i g_{n}(x)$ exists on $[0,1]$. Let $\varepsilon>0$, $\rho>0$, and $0<x_{1} \leqslant 1$ be given; we will find an $N$ such that $\left|S_{n}(x)\right|<\varepsilon$ for all $n \geqslant N$ if $x \in\left[x_{1}, 1\right]$ and $S_{n}(0)=c e^{i \theta}$ satisfies $c \in[0, \rho]$ and $\theta \in[0, \pi / 2]$. First ,we find an $N_{0}$ such that $\left|g_{n}(x)\right|<\varepsilon / \sqrt{2}$ for $x \in\left[x_{1}, 1\right]$ and $n \geqslant N_{0}$. We may assume $\varepsilon \leqslant x_{1} / \sqrt{2}$. We define $N_{0}$ via Lemma 2.5 using $E=[0,1], K=8 \rho / x_{1} \varepsilon^{2}$, and $\delta=x_{1} / 4$. Since $g_{n}$ is decreasing and nonnegative we will be done if we can show that $g_{n}\left(x_{1}\right)<\varepsilon / \sqrt{2}$ for all $n \geqslant N_{0}$. Suppose this were not the case. Then $g_{n}(x) \geqslant$ $\varepsilon / \sqrt{2}$ for some $n \geqslant N_{0}$ and for all $x \in\left[0, x_{1}\right]$. If $f_{n}(x)<g_{n}(x)$ for all $x \in[0, a)$, 
then $f_{n}^{\prime}(x)>1$ for $x \in[0, a)$ and hence $f_{n}(a)>a$. Thus, by Lemma $2.3, f_{n}(x) \geqslant$ $\min \left\{x, g_{n}(x)\right\}$ for $0 \leqslant x \leqslant 1$ and hence $g_{n}^{\prime}(x) \leqslant-2 A_{n}(x) \min \left\{x, g_{n}(x)\right\} g_{n}(x) \leqslant$ $-2 A_{n}(x) \min \left\{x_{1} / 2, \varepsilon / \sqrt{2}\right\} \varepsilon / \sqrt{2} \leqslant-\varepsilon^{2} A_{n}(x)$ for all $x \in\left[x_{1} / 2, x_{1}\right]$. Integrating, we find that

$$
g_{n}\left(x_{1}\right) \leqslant g_{n}\left(x_{1} / 2\right)-\varepsilon^{2} \int_{x_{1 / 2}}^{x_{1}} A_{n}(x) d x \leqslant c-\varepsilon^{2}\left(x_{1} / 4\right)\left(8 \rho / x_{1} \varepsilon^{2}\right)=c-2 \rho<0
$$

in contradiction to Lemma 2.2 .

Next we find $N$ such that $\left|f_{n}(x)\right|<\varepsilon / \sqrt{2}$ for $x \in\left[x_{1}, 1\right]$ and $n \geqslant N$. First use the foregoing to obtain an $N_{0}^{\prime}$ such that $\left|g_{n}(x)\right|<\varepsilon / 4 \sqrt{2}$ for $x \in\left[x_{1}-\varepsilon / 2 \sqrt{2}\right.$, 1] and for all $n \geqslant N_{0}^{\prime}$. We now use Lemma 2.5 with $K=128 \sqrt{2}(1+\rho) / 3 \varepsilon^{3}$ and $\delta=\varepsilon / 4 \sqrt{2}$ to find an $N \geqslant N_{0}^{\prime}$ such that $A_{n}(x)>K$ off a set of measure less than $\delta$ for all $n \geqslant N$. Now let $x_{0} \in\left[x_{1}, 1\right]$ and assume $f_{n}\left(x_{0}\right) \geqslant \varepsilon / \sqrt{2}$ for some $n \geqslant N$. Then $f_{n}(x)>$ $\varepsilon / 2 \sqrt{2}$ for $x_{0}-\varepsilon / 2 \sqrt{2}<x<x_{0}$ since $f_{n}^{\prime}(x)<1$ when $f_{n}(x)>g_{n}(x)$. Moreover, $f_{n}(x)^{2}-g_{n}(x)^{2}>\varepsilon^{2} / 8-\varepsilon^{2} / 32=3 \varepsilon^{2} / 32$ on $\left(x_{0}-\varepsilon / 2 \sqrt{2}, x_{0}\right)$ and therefore $f_{n}^{\prime}(x)$ $=1-A_{n}(x)\left[f_{n}(x)^{2}-g_{n}(x)^{2}\right]<1-3 \varepsilon^{2} A_{n}(x) / 32$. Integrating, we obtain

$$
f_{n}\left(x_{0}\right)<f_{n}\left(x_{0}-\varepsilon / 2 \sqrt{2}\right)+\varepsilon / 2 \sqrt{2}-\left(3 \varepsilon^{2} / 32\right) \int_{x_{0}-\varepsilon / 2 \sqrt{2}}^{x_{0}} A_{n}(x) d x
$$

which leads to

$$
\begin{aligned}
f_{n}\left(x_{0}-\varepsilon / 2 \sqrt{2}\right) & >\varepsilon / 2 \sqrt{2}+\left(3 \varepsilon^{2} / 32\right)(\varepsilon / 4 \sqrt{2}) 128 \sqrt{2}(1+\rho) / 3 \varepsilon^{3} \\
& =\varepsilon / 2 \sqrt{2}+1+\rho>1+c,
\end{aligned}
$$

contradicting Lemma 2.4. Hence, for $n \geqslant N$ we have $\left|S_{n}(x)\right|^{2}=f_{n}(x)^{2}+g_{n}(x)^{2}<\varepsilon^{2}$ for $x \in\left[x_{1}, 1\right]$.

There are two further points which we wish to discuss here. The first is the manner in which $S_{n}(x) \rightarrow 0$ in the complex plane. The requisite result is the following:

Proposition 2.7. Let $x_{1} \in(0,1]$ and $\varepsilon>0$ be given. Let $S_{n}(x)=f_{n}(x)+i g_{n}(x)$ and $A_{n}(x)$ be as above except that our initial condition is imposed at some $x_{0} \in$ $\left[0, x_{1} / 2\right]: S_{n}\left(x_{0}\right)=c e^{i \theta}$. Then there exists an integer $N$ such that, for all $n \geqslant N$, $\left|S_{n}(x)\right|<\varepsilon$ and $f_{n}(x)>g_{n}(x)$ for all $x \in\left[x_{1}, 1\right]$, for any choice of initial condition with $c \in[0, \rho]$ and $\theta \in[0, \pi / 2]$, and for any positioning of the initial condition within $\left[0, x_{1} / 2\right]$. Thus, $S_{n}(x) \rightarrow 0$ through the sector $\{z \in \mathbf{C} \mid 0 \leqslant \arg z<\pi / 4\}$ for all $x \in$ $(0,1]$ with uniformity over $x \in\left[x_{1}, 1\right]$ for $x_{1}>0$ and over initial data $c \in[0, \rho]$, $\theta \in[0, \pi / 2]$ imposed at $x_{0} \in\left[0, x_{1} / 2\right]$.

Proof. Again we use Lemma 2.5 to find $N$, this time for $K=128 \rho / x_{1}^{3}$ and $\delta=x_{1} / 8$. By Lemma 2.3, $f_{n}(x)>g_{n}(x)$ for all $n \geqslant N$ will hold on $\left[x_{1}, 1\right]$ if we can show that it holds for $x=x_{1}$. Suppose that for some $n \geqslant N$ we have $f_{n}\left(x_{1}\right) \leqslant g_{n}\left(x_{1}\right)$. Then Lemma 2.3 implies that $f_{n}(x)<g_{n}(x)$ for $x \in\left[0, x_{1}\right)$ and hence, by (2.2a), $f_{n}(x)>x_{1} / 4$ on $\left(3 x_{1} / 4, x_{1}\right)$ since $f_{n}\left(x_{1} / 2\right) \geqslant 0$. Thus $g_{n}^{\prime}(x) \leqslant-2\left(x_{1}^{2} / 16\right) A_{n}(x)$ on $\left(3 x_{1} / 4, x_{1}\right)$ and integration yields

$$
\begin{aligned}
g_{n}\left(x_{1}\right) & \leqslant g_{n}\left(3 x_{1} / 4\right)-2\left(x_{1}^{2} / 16\right) \int_{3 x_{1} / 4}^{x_{1}} A_{n}(x) d x \\
& \leqslant \rho-2\left(x_{1}^{2} / 16\right)\left(x_{1} / 8\right) 128 \rho / x_{1}^{3}=-\rho,
\end{aligned}
$$


contradicting Lemma 2.2. To conclude the proof we increase $N$ if necessary to insure that $\left|S_{n}(x)\right|<\varepsilon$ for all $n \geqslant N$ and $x \in\left[x_{1}, 1\right]$. That this can be done for $S_{n}(x)$ initialized at $x_{0} \in\left[0, x_{1} / 2\right]$ with $N$ independent of $x_{0}$ follows from the proof of Proposition 2.6.

Lastly, we wish to extend Proposition 2.6 to the case where $S_{n}(0)=c e^{i \theta}$ is allowed to lie in a larger sector. In particular, to be able to conclude anything about resonances we must be able to deal with values of $\theta$ in $[\pi / 2, \pi / 2+\varepsilon$ ) for some $\varepsilon>0$. In the following we shall allow $\theta \in[\pi / 2,5 \pi / 8]$. Note that with this initial condition $f_{n}(0)=c \cos \theta \leqslant 0$ and $g_{n}(0)=c \sin \theta \geqslant 0$. From equations (2.2a) and (2.2b), initially $f_{n}^{\prime} \geqslant 1$ and $g_{n}^{\prime} \geqslant 0$ and we see that this situation must prevail at least until $f_{n}$ reaches 0 . It is easy to see that $f_{n}$ and $g_{n}$ exist at least until $f_{n}$ reaches zero; from that point on we can invoke the previous results of this section to reach the desired conclusion. We begin by establishing two useful lemmas.

LEMMA 2.8. Let $S_{n}(x)=f_{n}(x)+i g_{n}(x)$ satisfy equation (2.3) with initial condition $S_{n}(0)=c e^{i \theta}$ where $c \geqslant 0$ and $\theta \in[\pi / 2,5 \pi / 8]$. Then for any $x \in(0,1], f_{n}(x)>0$ eventually. Thus, for $n$ large enough, $f_{n}$ has a unique zero $z_{n}$ in $[0,1]$.

Proof. Assume there is an $x_{1} \in(0,1]$ for which the conclusions of the lemma are false. Passing to a subsequence if necessary, we may assume $f_{n}\left(x_{1}\right) \leqslant 0$ for all $n$. By the proof of Lemma $2.1, f_{n}$ can have at most one zero, at which $f_{n}$ must necessarily cross the $x$-axis. Therefore, we must have $f_{n}(x)<0$ for $x \in\left[0, x_{1}\right)$ and thus also $g_{n}^{\prime}(x) \geqslant 0$ and $f_{n}^{\prime}(x) \geqslant 1$ on this interval. It then follows that

$$
\begin{aligned}
g_{n}(x)^{2}-f_{n}(x)^{2} & \geqslant g_{n}(0)^{2}-f_{n}(0)^{2}=c^{2}\left(\sin ^{2} \theta-\cos ^{2} \theta\right) \\
& =-c^{2} \cos 2 \theta>-c^{2} \cos 5 \pi / 4
\end{aligned}
$$

Thus,

$$
f_{n}^{\prime}(x) \geqslant 1+c^{2} A_{n}(x) / \sqrt{2}
$$

on $\left[0, x_{1}\right]$ or

$$
f_{n}\left(x_{1}\right) \geqslant f_{n}(0)+x_{1}+\left(c^{2} / \sqrt{2}\right) \int_{0}^{x_{1}} A_{n}(x) d x
$$

which yields a contradiction with $0 \geqslant f_{n}\left(x_{1}\right)$ by an appropriate application of Lemma 2.5.

LEMMA 2.9. With hypotheses as in the previous lemma, $g_{n}\left(z_{n}\right)=\max _{0 \leqslant x \leqslant 1} g_{n}(x)$ is bounded by a constant independent of $n$. Specifically, $g_{n}\left(z_{n}\right) \leqslant c \sqrt{1+1 / \sqrt{2}}$.

Proof. Note that $f_{n}(x)<0$ on $\left[0, z_{n}\right)$ and that $f_{n}^{\prime}$ and $g_{n}^{\prime}$ are nonnegative on this interval. Hence, $\left|f_{n}(x) / g_{n}(x)\right|$ is decreasing on $\left[0, z_{n}\right)$ so

$$
\begin{aligned}
\left|f_{n}(x) / g_{n}(x)\right| & \leqslant\left|f_{n}(0) / g_{n}(0)\right|=|\cot \theta| \\
& \leqslant|\cot 5 \pi / 8|=\sqrt{2}-1
\end{aligned}
$$




$$
\begin{aligned}
& \text { or }\left|f_{n}(x)\right| \leqslant(\sqrt{2}-1) g_{n}(x) \text {. Now } \\
& \qquad \begin{aligned}
g_{n}^{\prime}(x) & =2 A_{n}(x)\left|f_{n}(x)\right| g_{n}(x) \leqslant 2(\sqrt{2}-1) A_{n}(x) g_{n}(x)^{2} \\
& =A_{n}(x) g_{n}(x)^{2}\left[1-(\sqrt{2}-1)^{2}\right] \leqslant A_{n}(x)\left[g_{n}(x)^{2}-f_{n}(x)^{2}\right] \\
& <1-A_{n}(x)\left[f_{n}(x)^{2}-g_{n}(x)^{2}\right]=f_{n}^{\prime}(x) .
\end{aligned}
\end{aligned}
$$

Hence,

$$
\begin{aligned}
g_{n}\left(z_{n}\right) & \leqslant g_{n}(0)+f_{n}\left(z_{n}\right)-f_{n}(0)=c \sin \theta-c \cos \theta \\
& <c(\sin 5 \pi / 8-\cos 5 \pi / 8)=c \sqrt{1+1 \sqrt{2}} .
\end{aligned}
$$

These lemmas allow us to carry out the argument outlined above to establish

THEOREM 2.10. Let $S_{n}(x)=f_{n}(x)+i g_{n}(x)$ satisfy equation (2.3) where $\left\{A_{n}(x)\right\}$ is as described following equation (2.3). Set $B_{\rho}=\{z \in C|0 \leqslant \arg z \leqslant 5 \pi / 8| z \mid, \leqslant \rho\}$. Then $S_{n}(x)$ exists on $[0,1]$ and $S_{n}(x) \rightarrow 0$ as $n \rightarrow \infty$ uniformly with respect to $S_{n}(0) \in B_{\rho}$ and $x \in\left[x_{1}, 1\right]$ for any $\rho>0$ and any $x_{1}>0$. In addition, for any $x_{1}>0$ there exists an integer $N$ such that $0 \leqslant \arg S_{n}(x)<\pi / 4$ for all $n \geqslant N$ and all $x \in\left[x_{1}, 1\right]$.

Proof. We can restrict our attention to $\theta \in[\pi / 2,5 \pi / 8]$ since Proposition 2.7 establishes the result for $\theta \in[0, \pi / 2]$. We observed just before Lemma 2.8 that $S_{n}(x)$ exists on [0,1]. Lastly, we fix $x_{1}>0$ and look at the behavior of $S_{n}(x)$ for $x \in\left[x_{1}, 1\right]$. By Lemma 2.8 we can find an integer $N_{0}$ such that $f_{n}\left(x_{1} / 2\right)>0$ for all $n \geqslant N_{0}$ and thus $z_{n}<x_{1} / 2$ for all $n \geqslant N_{0}$. We also know that $g_{n}\left(z_{n}\right)<c \sqrt{1+1 / \sqrt{2}}$ $\leqslant \rho \sqrt{1+1 / \sqrt{2}}$. We now view $S_{n}(x)$ as defined by an initial condition at $x_{0}=z_{n}$. Then by Proposition 2.7 (with $\rho$ replaced by $\rho \sqrt{1+1 / \sqrt{2}}$ ), given $\varepsilon>0$, we can find an integer $N \geqslant N_{0}$ such that for all $n \geqslant N$ we have $\left|S_{n}(x)\right|<\varepsilon$ and $0 \leqslant \arg S_{n}(x)<$ $\varepsilon / 4$ for all $x \in\left[x_{1}, 1\right]$. Since this $N$ depends only on $\rho, x_{1}$ and $\varepsilon$, the stated uniform convergence follows immediately.

REMARK. Analogous results hold for $S_{n}(x)$ lying in the lower half-plane since equation (2.3) is unchanged in form under $S_{n} \rightarrow \bar{S}_{n}$.

3. The comparison argument. To deal effectively with the resonance problem we must have results of the type of Theorem 2.10 that apply to the Riccati equation (2.1) with $A_{n}(x)$ replaced by $A_{n}(x)-E, E \in \mathrm{C}$. We do this using a comparison argument based in spirit on Harrell's argument in [21]. To fix notation we shall consider

$$
\begin{aligned}
& u_{n}^{\prime \prime}(x)=A_{n}(x) u_{n}(x), \\
& v_{n}^{\prime \prime}(x)=\left(A_{n}(x)-E\right) v_{n}(x)
\end{aligned}
$$

on $[0,1]$ with $u_{n}(0)=1=v_{n}(0)$ and $u_{n}^{\prime}(0)=c^{-1} e^{-i \theta}=v_{n}^{\prime}(0)$ where $c>0$ and $\theta \in$ $(0,5 \pi / 8]$. Then the function $S_{n}(x)=u_{n}(x) / u_{n}^{\prime}(x)$ satisfies $S_{n}^{\prime}(x)=1-$ $A_{n}(x)\left[S_{n}(x)\right]^{2}$ with initial condition $S_{n}(0)=c e^{i \theta}$ which conforms with our notation 
in §2. Our comparison result gives information about the behavior of $v_{n}(x)$ through a study of the function $w_{n}(x)$ which relates $u_{n}(x)$ and $v_{n}(x)$ via

$$
v_{n}(x)=w_{n}(x) u_{n}(x) \text {. }
$$

The major result in this section is

Theorem 3.1. Suppose $R>0,0<\sqrt{(1-1 / \sqrt{2}) / 2 R} \leqslant \rho_{1} \leqslant \rho_{2}$, and $A_{n}(x), n=$ $1,2,3, \ldots$, are real-valued integrable functions on $[0,1]$ with $A_{n}(x) \geqslant 0$ and $A_{n}(x) \rightarrow \infty$ as $n \rightarrow \infty$ almost everywhere in $[0,1]$. Define $B_{\rho_{1}, \rho_{2}}=\{z \in \mathbf{C} \mid 0<\arg z \leqslant 5 \pi / 8$, $\left.\rho_{1} \leqslant|z| \leqslant \rho_{2}\right\}$. Then for any $x_{1} \in(0,1]$ and $\varepsilon>0$ there exists an integer $N$ with the following properties: If $v_{n}(x)$ satisfies equation (3.2) with $|E| \leqslant R$ and has initial conditions $v_{n}(0)=1, v_{n}^{\prime}(0)=c^{-1} e^{-i \theta}$ where ce $e^{i \theta} \in B_{\rho_{1}, \rho_{2}}$, then $v_{n}(x) / v_{n}^{\prime}(x)<\varepsilon$ for all $x \in\left[x_{1}, 1\right]$ for all $n \geqslant N$. In particular, $v_{n}(1) / v_{n}^{\prime}(1) \rightarrow 0$ uniformly as $n \rightarrow \infty$ for all $E$ contained in any compact subset of $\mathbf{C}$ and for all initial conditions in $B_{\rho_{1}, \rho_{2}}$.

Proof. We shall have recourse to two comparison arguments which are instrumental in showing that $v_{n}(x) / v_{n}^{\prime}(x)$ has the same behavior as does $S_{n}(x)$ of Theorem 2.10. For the further development of these results we introduce the auxiliary function

$$
\Omega_{n}(x)=w_{n}^{\prime}(x) / w_{n}(x)
$$

where $w_{n}(x)$ is defined by equation (3.3) above. That $w_{n}(x)$ is defined by this relation is guaranteed by the argument used to prove Lemma 2.2 which tells us that $g_{n}(x)$, and hence $u_{n}(x)$, is never zero on $[0,1]$ when $c>0$ and $\theta \in(0,5 \pi / 8]$. However, justification of our definition of $\Omega_{n}(x)$ will be made only in the course of the main argument where it will be shown that $\left|\Omega_{n}(x)\right|$ obeys a differential inequality which forces it to remain bounded on $[0,1]$. For now, we note that from the initial conditions for $u_{n}$ and $v_{n}$ we must have $w_{n}(0)=1$ and $w_{n}^{\prime}(0)=0$ so that $\Omega_{n}(x)$ is certainly defined and continuous for positive $x$ near zero. On this interval $\Omega_{n}(x)$ satisfies

$$
\Omega_{n}^{\prime}(x)=-E-2\left[u_{n}^{\prime}(x) / u_{n}(x)\right] \Omega_{n}(x)-\left[\Omega_{n}(x)\right]^{2}
$$

with initial condition $\Omega_{n}(0)=0$ and $|E| \leqslant R$.

It is straightforward to show (we suppress the subscript $n$ in the following)

$$
\frac{1}{2} \frac{d}{d x}|\Omega(x)|^{2}=-\operatorname{Re}[E \bar{\Omega}(x)]-2\left[\operatorname{Re} u^{\prime}(x) / u(x)\right]|\Omega(x)|^{2}-|\Omega(x)|^{2} \operatorname{Re} \Omega(x) .
$$

It then follows that, for $|\Omega(x)| \neq 0$,

$$
\frac{d}{d x}|\Omega(x)| \leqslant R-2\left[\operatorname{Re} u^{\prime}(x) / u(x)\right]|\Omega(x)|+|\Omega(x)|^{2} .
$$

Now let $G(x)$ be defined by

$$
G^{\prime}(x)=R-2\left[\operatorname{Re} u^{\prime}(x) / u(x)\right] G(x)+[G(x)]^{2}
$$


with initial condition $G(0)=0$. We use a comparison argument to show that $G(x) \geqslant|\Omega(x)|$ for $x$ in the domain of definition of $G(x)$. First note that $G^{\prime}(x)=R$ $>0$ at any zero of $G$ so we have $G(x)>0$ for $x>0$. To establish that $G(x) \geqslant|\Omega(x)|$ we consider the family of functions $G_{\delta}(x), \delta>0$, defined by the differential equations

$$
G_{\delta}^{\prime}(x)=R+\delta-2\left[\operatorname{Re} u^{\prime}(x) / u(x)\right] G_{\delta}(x)+G_{\delta}(x)^{2}
$$

with initial conditions $G_{\delta}(0)=\delta$. Then $G_{\delta}(0)>|\Omega(0)|=0$ and hence $G_{\delta}(x)>0$ for $x>0$ by the same argument as was given for $G$ above. We claim $G_{\delta}(x)>|\Omega(x)|$ for $x \geqslant 0$. For suppose this were not so. Then there would be an $x_{0}>0$ such that $G_{\delta}\left(x_{0}\right)=\left|\Omega\left(x_{0}\right)\right|$ and $G_{\delta}(x)>|\Omega(x)|$ for all $x \in\left[0, x_{0}\right)$. Then $\left|\Omega\left(x_{0}\right)\right| \neq 0$ and by our differential inequality we must have

$$
\frac{d}{d x}|\Omega(x)|_{x=x_{0}} \leqslant R-2\left[\operatorname{Re} \frac{u^{\prime}\left(x_{0}\right)}{u\left(x_{0}\right)}\right] G_{\delta}\left(x_{0}\right)+\left[G_{\delta}\left(x_{0}\right)\right]^{2}<G_{\delta}^{\prime}\left(x_{0}\right) .
$$

However, since $G_{\delta}(x)>|\Omega(x)|$ to the left of $x_{0}$ we must have

$$
G_{\delta}^{\prime}\left(x_{0}\right) \leqslant\left.\frac{d}{d x}|\Omega(x)|\right|_{x=x_{0}}
$$

and we have a contradiction. Thus, $G_{\delta}(x)>|\Omega(x)|$ for all $x$ in the domain of definition of $G_{\delta}(x)$. Finally, we take the limit $\delta \downarrow 0$ to conclude that $G(x) \geqslant|\Omega(x)|$. Here we have used the fact that solutions to a differential equation are continuous functions of initial conditions and of parameters appearing in the equation itself - for precise statements see [17].

We now wish to show that $G(x)=G_{n}(x)$ exists on $[0,1]$ if $n$ is large enough. We accomplish this via another comparison argument. First we must examine the behavior of the coefficient

$$
\operatorname{Re} \frac{u_{n}^{\prime}(x)}{u_{n}(x)}=\frac{f_{n}(x)}{f_{n}(x)^{2}+g_{n}(x)^{2}}=\operatorname{Re}\left[S_{n}(x)^{-1}\right] .
$$

Now by Theorem 2.10 for any $\varepsilon>0$ and any $x_{1} \in(0,1]$, there is an integer $N$ such that $\left|S_{n}(x)\right|<\varepsilon$ and $0<\arg S_{n}(x)<\pi / 4$ for $n \geqslant N$ and $x \in\left[x_{1}, 1\right]$. Now $g_{n}(x)$ is never zero, hence $S_{n}(x)$ is never 0 for $c>0$ and $\theta \in(0,5 \pi / 8]$. This implies that $S_{n}(x)^{-1} \rightarrow \infty$ in the sector $\{z \in \mathbf{C} \mid 0>\arg z>-\pi / 4\}$ uniformly on $\left[x_{1}, 1\right]$, from which it follows that $\operatorname{Re}\left[S_{n}(x)^{-1}\right] \rightarrow \infty$ uniformly for $x$ in the given interval. Furthermore, we can bound $\operatorname{Re}\left[S_{n}(x)^{-1}\right]$ below by the constant $c^{-1} \cos 5 \pi / 8=$ $-c^{-1} \sqrt{(1-1 / \sqrt{2}) / 2}$ on the entire interval from 0 to 1 . To see this, one notes that we need only consider the interval on which $f_{n}<0$ and there both $f_{n}$ and $g_{n}$ are increasing. Then

$$
\begin{aligned}
\operatorname{Re} \frac{u_{n}^{\prime}(x)}{u_{n}(x)} & =\frac{f_{n}(x)}{f_{n}(x)^{2}+g_{n}(x)^{2}} \geqslant \frac{f_{n}(x)}{f_{n}(x)^{2}+g_{n}(0)^{2}} \\
& \geqslant \frac{f_{n}(0)}{f_{n}(0)^{2}+g_{n}(0)^{2}} \geqslant c^{-1} \cos \frac{5 \pi}{8}=-c^{-1} \sqrt{\frac{1}{2}(1-1 / \sqrt{2})}
\end{aligned}
$$

because the function $h(t)=t /\left(t^{2}+g_{n}(0)^{2}\right)$ is increasing when $|t| \leqslant g_{n}(0)$. 
We now define the function

$$
\Phi(x)= \begin{cases}-\sqrt{R} & \text { for } 0 \leqslant x \leqslant(3 \sqrt{R})^{-1}, \\ \sqrt{R} & \text { for }(3 \sqrt{R})^{-1}<x \leqslant 1,\end{cases}
$$

where $R$ is the bound on the allowed energies which appears in equation (3.7). We claim that $\Phi(x) \leqslant \operatorname{Re}\left[u_{n}^{\prime}(x) / u_{n}(x)\right]$ on $[0,1]$ for $n$ large enough. If we take $x_{1}=$ $(3 \sqrt{R})^{-1}$, then by our discussion in the preceding paragraph we can find an integer $N$ such that $\operatorname{Re}\left[u_{n}^{\prime}(x) / u_{n}(x)\right]>\sqrt{R}$ for all $n \geqslant N$, thus establishing the claim for $x \in\left((3 \sqrt{R})^{-1}, 1\right]$. The claim also holds on $\left[0,(3 \sqrt{R})^{-1}\right]$ if $c \in B_{\rho_{1}, \rho_{2}}$ by the stated restriction on $\rho_{1}$.

We now introduce our second comparison equation

$$
H^{\prime}(x)=R-2 \Phi(x) H(x)+H(x)^{2}
$$

with the initial condition $H(0)=0$. This equation can be solved explicitly:

$$
H(x)= \begin{cases}\frac{R x}{1-\sqrt{R} x} & \text { for } 0 \leqslant x \leqslant \frac{1}{3 \sqrt{R}} \\ \frac{3 R x+2 \sqrt{R}}{3 \sqrt{R} x+5} & \text { for } \frac{1}{3 \sqrt{R}} \leqslant x \leqslant 1\end{cases}
$$

Since equation (3.10) thus has a solution on $[0,1]$ for initial data 0 , by an elementary comparison argument (see, e.g. [22]) the equation (3.5) must also, and thus the existence of $G(x)$ on the interval $[0,1]$ is established. Hence, the existence and boundedness of $\Omega_{n}(x)$ for sufficiently large $n$ is established as well. This justifies our definition of $\Omega_{n}(x)$ by equation (3.4) and shows that $w_{n}(x) \neq 0$ and hence $v_{n}(x) \neq 0$ for all $x \in[0,1]$.

We now complete the proof of the theorem. From equation (3.3) we obtain

$$
\frac{v_{n}^{\prime}(x)}{v_{n}(x)}=\frac{u_{n}^{\prime}(x)}{u_{n}(x)}+\frac{w_{n}^{\prime}(x)}{w_{n}(x)}=\frac{1}{S_{n}(x)}+\Omega_{n}(x) .
$$

From the above, we know that $\Omega_{n}(x)$ is bounded on $[0,1]$ independently of $n$ for large $n$, of $c e^{i \theta}$ for $c e^{i \theta} \in B_{\rho_{1}, \rho_{2}} \equiv\left\{c e^{i \theta} \in \mathbf{C} \mid 0<\theta \leqslant 5 \pi / 8, \rho_{1} \leqslant c \leqslant \rho_{2}\right\}$, and of $E$ with $|E| \leqslant R$ where $R \geqslant(1-1 / \sqrt{2}) / 2 \rho_{1}^{2}$. Since $S_{n}(x) \rightarrow 0$ uniformly for such sets as well as on the sets $\left[x_{1}, 1\right]$ where $x_{1}>0$, we must have $v_{n}^{\prime}(x) / v_{n}(x) \rightarrow \infty$ uniformly for all these sets. Equivalently, $v_{n}(x) / v_{n}^{\prime}(x) \rightarrow 0$ uniformly for the aforementioned sets. In particular, $v_{n}(1) / v_{n}^{\prime}(1) \rightarrow 0$ uniformly for $|E| \leqslant R$ and for initial values $c e^{i \theta}$ of $v_{n}(x) / v_{n}^{\prime}(x)$ in $B_{\rho_{1}, \rho_{2}}$.

4. The main result. As mentioned in the Introduction, we have only to combine the results of the previous sections with Hurwitz's Theorem to prove stability of resonances. We first state this theorem. 
THEOREM 4.1 (HuRWITZ'S THEOREM). Let $\left\{F_{n}\right\}$ be a sequence of analytic functions on a domain $D$ with $F_{n} \rightarrow F$ uniformly on compact subsets of $D$. Let $\overline{B(a, R)} \equiv\{z \in$ $C \| z-a \mid \leqslant R\} \subset D$ and suppose $F$ has no zeros on $\{z|| z-a \mid=R\}$ and $k$ zeros on $B(a, R) \equiv\{z \in C|| z-a \mid<R\}$. Then there is an integer $N$ such that, for all $n \geqslant N$, $F_{n}$ has exactly $k$ zeros on $B(a, R)$. (Zeros are counted according to their multiplicity.)

Proof. See Conway [19] or Duncan [20].

CoROllaRY 4.2. Let $\left\{F_{n}\right\}$ and $F$ be as above with $F \neq 0$. Then $\tilde{z} \in D$ is a zero of $F$ if and only if $\tilde{z}=\lim _{n \rightarrow \infty} z_{n}$ where, for each $n, z_{n}$ is a zero of the function $F_{n}$.

For us $F_{n}(E)=\psi_{0}(1 ; E) / \psi_{0}^{\prime}(1 ; E)-\psi_{\infty}(1 ; E, n) / \psi_{\infty}^{\prime}(1 ; E, n)$ defines our sequence of functions and we wish to show that the second term goes to zero as $n$ goes to infinity uniformly for $E$ in compact sets. This will follow from Theorem 3.1 once we translate the equation and conditions for $\psi_{\infty}$ on the interval $[1, a]$ into the corresponding equation and conditions on $[0,1]$. To do this we set

$$
t=(a-x) /(a-1) \text { and } v_{n}(t)=\psi_{\infty}(x ; E, n) .
$$

We find that $\psi_{\infty}(1 ; E, n) / \psi_{\infty}^{\prime}(1 ; E, n)=-(a-1) v_{n}(1) / v_{n}^{\prime}(1)$ where $v_{n}$ satisfies equation (3.2) with $E$ replaced by $(a-1)^{2} E$, with initial condition $v_{n}(0) / v_{n}^{\prime}(0)=$ $i /[(a-1) \sqrt{E}]$, and with $A_{n}(x)=(a-1)^{2} W_{n}(a-(a-1) x)$. For convenience we set $\tilde{E}=(a-1)^{2} E$. Then, relating our initial data to the variables appearing in Theorem 3.1, we have $c e^{i \theta}=i(\tilde{E})^{-1 / 2}$ or $c=(|\tilde{E}|)^{-1 / 2}$ and $\theta=\frac{\pi}{2}-\frac{1}{2} \arg \tilde{E}$. Thus, Theorem 3.1 applies for $\arg E=\arg \tilde{E} \in[-\pi / 4, \pi)$ and $\rho_{1} \leqslant(|\tilde{E}|)^{-1 / 2} \leqslant \rho_{2}$ where $\rho_{1}>0$, and we may conclude that $v_{n}(1) / v_{n}^{\prime}(1) \rightarrow 0$ uniformly as $n \rightarrow \infty$ for $\tilde{E}$ (and hence also $E)$ in annular sectors. This implies $\psi_{\infty}(1 ; E, n) / \psi_{\infty}^{\prime}(1 ; E, n) \rightarrow 0$ uniformly as $n \rightarrow \infty$ for $E$ in sets of the form $\left\{z \in \mathrm{C}\left|-\pi / 4 \leqslant \arg z<\pi, R_{1} \leqslant\right| z \mid \leqslant R_{2}\right\}$ where $R_{1}>0$ and $R_{2}$ is finite.

Now Hurwitz's Theorem can be applied to yield the following stability of resonances result.

THEOREM 4.3. Given any unperturbed eigenvalue $e>0$ and a ball $B(e ; \varepsilon) \equiv$ $\{z \in \mathbf{C}|| z-e \mid<\varepsilon\} \subset\{z \in \mathbf{C}|| z \mid>0,-\pi / 4<\arg z<\pi\}$ which contains no other unperturbed eigenvalue, for all $n$ sufficiently large there is a unique resonance $E_{n}$ of the perturbed problem of index $n$ with $E_{n}$ converging to $e$ as $n \rightarrow \infty$. Furthermore, the values $e>0$ are the only limit points of solutions to $F_{n}(E)=0$ as $n \rightarrow \infty$ which lie in the open sector $\{E \in \mathbf{C}|| E \mid>0,-\pi / 4<\arg E<\pi\}$. In addition, all resonances lie below the real axis.

Proof. That the zeros of $\psi_{0}(1 ; E)$ occur on the real axis and are simple follows from the fact that these zeros are the eigenvalues of the regular Sturm-Liouville problem with unmixed boundary conditions given by equation (1.3). The functions $F_{n}(E)$ and $F(E)=\psi_{0}(1 ; E) / \psi_{0}^{\prime}(1 ; E)$ have poles at the zeros of $\psi_{0}^{\prime}(1 ; E)$-note that $\psi_{\infty} / \psi_{\infty}^{\prime} \rightarrow 0$ with $n$ going to infinity by Theorem 3.1 , so that there cannot be "cancelling poles" in $\psi_{\infty} / \psi_{\infty}^{\prime}$ for large enough $n$. Draw disks around these poles so small that no zeros of $F$ or $F_{n}$ lie within them. Zeros of $\psi_{0}$ and $\psi_{0}^{\prime}$ cannot coincide since $\psi_{0}$ is not the zero solution. Hurwitz's Theorem can now be applied to the sector with these disks excised. 
That resonances cannot lie on the positive real axis follows from observing that for real $E$ the real solution $\psi_{0}(x ; E)$ cannot be dependent with the inherently complex solution $\psi_{\infty}(x ; E, n)$. Finally, resonance energies have negative imaginary parts since a resonance with a positive imaginary part would provide an $L^{2}$ solution to Schrödinger's equation and hence the resonance would be an eigenvalue. But the operator associated with our problem is selfadjoint and cannot have a nonreal eigenvalue.

5. Concluding remarks. By developing the remark at the end of $\S 2$ one obtains information about the analytic continuations of $F_{n}(E)$ and $F(E)$ to the sector $\pi \leqslant \arg E \leqslant 9 \pi / 4$. For $\arg E=\pi$ or equivalently $\arg S_{n}(0)=0$, the results of $\S 3$ follow upon noting that, by Lemma $2.1, f_{n}(x) \neq 0$ for $x>0$ and thus $S_{n}(x) \neq 0$ for $x>0$. The last argument given above extends to show that resonance energies cannot satisfy $\pi<\arg E<2 \pi$ (by analytic continuation our convention on $\sqrt{E}$ for this region must be $\arg \sqrt{E}=\frac{1}{2} \arg E$ ). Thus any zeros of $F_{n}(E)$ with $0<\arg E<2 \pi$ must lie on the negative real axis and must be eigenvalues. Hurwitz's Theorem then shows that for any unperturbed eigenvalue $e<0$ we can find a sequence of perturbed eigenvalues $\left\{E_{n}\right\}$ with $F_{n}\left(E_{n}\right)=0$ and $E_{n} \rightarrow e$ as $n \rightarrow \infty$. The existence of perturbed eigenvalues corresponding to the negative unperturbed eigenvalues is also readily established using the min-max principle $[9,18]$. From that vantage point one also finds that the perturbed eigenvalues are less than their unperturbed counterparts. In addition, the min-max principle shows that if 0 is an unperturbed eigenvalue then there will be a sequence of corresponding perturbed eigenvalues which are less than 0 . However, it would be relatively difficult to prove convergence, $E_{n} \rightarrow e$, by this method in the generality discussed here. Thus our stability result may yield new, though by no means unexpected, results for eigenvalues.

In the parlance of theoretical physics the values of $E$ for which $0<\arg E<2 \pi$ constitute the "physical sheet" and our discussion above regards the well-known fact that resonances lie on the "second sheet" gotten by analytically continuing across the positive real axis from above to below. The complex conjugates of our resonances, which are zeros of the analytic continuation of $F_{n}(E)$ to $2 \pi<\arg E<9 \pi / 4$, give "incoming wave" solutions to Schrödinger's equation and are thus important to an understanding of scattering in the $t \rightarrow-\infty$ limit; put differently, they are resonances in the time-reversed setting.

\section{REFERENCES}

1. G. Gamow, Constitution of atomic nuclei and radioactivity, Oxford Univ. Press, Oxford, 1931.

2. A. J. F. Siegert, On the derivation of the dispersion formula for nuclear reactions, Phys. Rev. 56 (1939), $750-752$.

3. M. S. Ashbaugh and E. M. Harrell, Perturbation theory for shape resonances and large barrier potentials, Comm. Math. Phys. 83 (1982), 151-170.

4. E. Schrodinger, Quantisierung als Eigenwertproblem. IV: Störungstheorie mit Anwendung auf den Starkeffekt der Balmerlinien, Ann. Physik (7) 80 (1926), 437-490.

5. F. Rellich, Störungtheorie der Spektralzerlegung. I-V, Math. Ann. 113 (1937), 600-619, 667-685; 116 (1939), 555-570; 117 (1940), 356-382; 118 (1942), 462-484.

6. T. Kato, On the convergence of the perturbation method. I, II, Progr. Theoret. Phys. 4 (1949), 514-523; 5 (1950), 95-101, 207-212. 
7. B. Sz.-Nagy, Perturbations des transformations autoadjointes dans l'espace de Hilbert, Comment. Math. Helv. 19 (1946/47), 347-366.

8. B. Simon, Resonances in n-body quantum systems with dilatation analytic potentials and the foundations of time-dependent perturbation theory, Ann. of Math. (2) 97 (1973), 247-274.

9. M. Reed and B. Simon, Methods of modern mathamtical physics. Vol. IV: Analysis of operators, Academic Press, New York, 1978.

10. B. Simon, Resonances and complex scaling: a rigorous overview, Internat. J. Quantum. Chem. 14 (1978), 529-542.

11. H. Baumgärtel and M. Demuth, Decoupling by a projection, Rep. Math. Phys. 15 (1979), 173-186.

12. J. M. Combes, P. Duclos and R. Seiler, Krein's formula and one-dimensional multiple wells (to appear).

13. G. C. Emch and K. B. Sinha, Weak quantization in a nonperturbative model, J. Math. Phys. 20 (1979), 1336-1340.

14. B. Simon, Exterior complex scaling and molecular resonances in the Born-Oppenheimer approximation (to appear).

15. E. Vock and W. Hunziker, Stability of Schrödinger eigenvalue problems, Comm. Math. Phys. 83 (1982), 281-302.

16. A. Böhm, Resonance poles and Gamow vectors in the rigged Hilbert space formulation of quantum mechanics (to appear).

17. E. A. Coddington and N. Levinson, Theory of ordinary differential equations, McGraw-Hill, New York, 1955.

18. M. S. Ashbaugh, Asymptotic perturbation theory for the eigenvalues of Schrödinger operators in a strong coupling limit, Thesis, Princeton Univ., Princeton, N. J., 1980.

19. J. Conway, Functions of one complex variable, Springer-Verlag, New York, 1973.

20. J. Duncan, The elements of complex analysis, Wiley, London, 1968.

21. E. M. Harrell, On the rate of asymptotic eigenvalue degeneracy, Comm. Math. Phys. 60 (1978), 73-95.

22. G. Birkhoff and G.-C. Rota, Ordinary differential equations, 3rd ed., Wiley, New York, 1978.

23. H. L. Roydzn, Real analysis, 2nd ed., Macmillan, New York, 1968.

24. S. Albeverio and R. Høegh-Krohn, Perturbation of resonances in quantum mechanics, J. Math. Anal. Appl. (to appear).

Department of Mathematics, University of Tennessee, Knoxville, Tennessee 37996-1300 (Current address of Carl Sundberg)

Current address (M. S. Ashbaugh): Department of Mathematics, University of Missouri, Columbia, Missouri 65211 